\section{Omnidirectional increase in threshold for image shifts during saccadic eye movements}

\author{
BRUCE BRIDGEMAN \\ Psychology Board of Studies, University of California, \\ Santa Cruz, California 95064 \\ and \\ LAWRENCE STARK \\ Department of Physiological Optics, \\ University of California, Berkeley, California 94720
}

The visual system must maintain a stable metric of visual space despite changes in the retinal position of the world during eye movements. To meet this challenge, there must be a mechanism for differentiating retinal shifts due to eye movement from shifts due to the displacement of objects in the world. Whipple and Wallach (1978) have termed this an "accounting" mechanism, while we have previously discussed it as a frame-of-reference computation (Stark, Kong, Schwartz, Hendry, \& Bridgeman, 1976). This mechanism becomes less sensitive during target displacements due to saccades, an effect which has been analyzed experimentally as a saccadic suppression of displacement. The failure of subjects to detect target movements occurring during eye movements, then, provides a tool for investigating the mechanism which accomplishes subjective stabilization.

The recent paper of Whipple and Wallach in Perception \& Psychophysics (1978) apparently comes from a less-well-instrumented era and contradicts the findings of other workers in this area. In addition to the data of Mack (1970) and of Wallach and Lewis (1966), which Whipple and Wallach cite, at least five other papers have appeared on this subject (Beeler, 1967; Brune \& Lücking, 1969; Bridgeman, Hendry, \& Stark, 1975; Mack, Fendrich, \& Pleune, 1978; Stark et al., 1976). Despite wide differences in their target parameters, dynamics of target movement, methods of eye-movement monitoring, conditions of saccade initiation, and mode of report, all of these papers reveal a much higher threshold for displacement detection when target and eye motion are congruent than the threshold found by Whipple and Wallach. This is especially surprising because Whipple and Wallach used a measure of perceived direction as well

Supported by NIH National Eye Institute Grant R01 EY01482 to B. Bridgeman. We thank $W$. Whipple for providing supplementary information used in this report, and $M$. Nagle, D. Bridgeman, and M. Mayer for careful reading of the manuscript. as displacement, and presumably subjects would have to know that a displacement had taken place before being able to accurately report its direction. Whipple and Wallach also interpret their data as showing a substantial difference between displacement thresholds in congruent and orthogonal target-movement conditions, in contrast to the data of Mack (1970) and Stark et al. (1976). We have resolved these discrepancies and show here that within the limitations of their techniques, Whipple and Wallach's results conform rather closely to other results showing higher threshold-displacement ratios and no difference between congruent and orthogonal displacements.

Many of the reasons for these discrepancies relate to technical features of the experimental apparatus. First, Whipple and Wallach measured vertical eye movements by monitoring the position of the lid of one eye, a method prone to large errors because of the unreliable correlation of lid position with vertical eye position. Even when these two positions correspond, the dynamics of lid movement and eye movement are different, so that the vertical components of Whipple and Wallach's target movements, driven by the eyelid recording, can be expected to differ from the actual eye movements.

The technique of measuring vertical movements in one eye and horizontal movements in the other raises a second question about the accuracy of the recording method. Measuring one dimension in each eye takes advantage of Hering's law of the equal innervation of the eyes (Hering, 1868), and is valid for registration of resting eye position. Dynamic violations of Hering's law are common and can be quite large, however (Bahill, Ciuffreda, Kenyon, \& Stark, 1976), and the vertical and horizontal components of a saccade may occur at different times with little overlap (Bahill \& Stark, 1975, 1977). These factors may have contributed to the uninterpretable data in Whipple and Wallach's oblique-oblique condition.

A third source of error in eye-movement recording which might reduce the observed displacement thresholds is the low-pass filter in the Biometrics amplifiers used by Whipple and Wallach. This filter, designed to reduce $60-\mathrm{Hz}$ powerline artifact, increases the time constant of the machine from $2 \mathrm{msec}$, fast enough to deliver a nearly undistorted record of a saccade, to $16 \mathrm{msec}$, slow enough to yield a perceptually significant slowing of the settling time. Thus, after a saccade ends, a stimulus driven by the filtered amplifier would continue an exponentially decrementing movement at a time when the saccadic suppression curve for displacements (Bridgeman, et al., 1975; Stark, et al., 1976) has returned nearly to baseline intersaccadic values. Whipple (Note 1) has verified that the Biometrics filters were used on some 
trials, and these, when averaged with unfiltered trials, would yield artifically reduced displacement thresholds.

The effects of these difficulties are minimized under conditions where the target is driven by a horizontal eye movement, and where the differences in threshold rather than their absolute values are considered. The problem reduces to explaining the cause of the threshold differences between Whipple and Wallach's Condition 5 (congruent movement, threshold $8 \%$ ) and their Conditions 4 and 6 (noncongruent movement, thresholds $25 \%$ and $24 \%$, respectively). Whipple and Wallach discuss these results in terms of a vector summation of eye motion and target motion, where the target is treated as the point on their $7^{\circ}$ circular stimulus which is fixated at the start of the trial. Their geometrically elegant analysis, however, does not take into account the likelihood that detection of displacement depends not on the position of the originally fixated point on the circle but on the part of the circle nearest the fovea at the end of the trial, when the subject's decision about target movement is made. When the data are reanalyzed in these terms, the difference between the congruent and the orthogonal conditions nearly disappears.

The following analysis assumes that detection of target displacement is limited by the final position of the part of the target nearest the fovea, where acuity is best and motion thresholds (Leibowitz, Johnson, \& Isabelle, 1972) are lowest. Consider first the case of horizontal eye movement and horizontal target movement (Whipple and Wallach's Condition 5): The eye begins on the left margin of the stimulus circle and jumps $7^{\circ}$ to the right side. At the same time, the stimulus moves (at threshold) by $8 \%$ of that amount, so that the discrepancy between the "expected" final position of the right side of the stimulus and its real position is $.08 \times 7^{\circ}$, or $.56^{\circ}$. For horizontal eye movement and vertical target movement (Whipple and Wallach's Condition 4), the situation is more complicated, because the shape of the stimulus influences the position and orientation of the contour nearest the fovea. The final position of this contour can be found with simple trigonometry (see Figure 1) by solving the triangle $C_{1} C_{2} F_{2}$, defined by the presaccadic center of the stimulus circle, the postsaccadic center, and the final eye position (which would have coincided with the edge of the target if it had not moved). The radius of the stimulus circle is then subtracted from the hypotenuse of the triangle, and the tangent of the circle at the resulting point is perpendicular to the hypotenuse. At Whipple and Wallach's threshold displacement ratio of $25 \%$, the linear distance from the final eye position to the nearest point on the stimulus circle is $.41^{\circ}$ at the end of the trial and the tangent of the circle at that point is inclined $26.5^{\circ}$ from the vertical $\left(\angle C_{1} F_{2} C_{2}\right.$ in

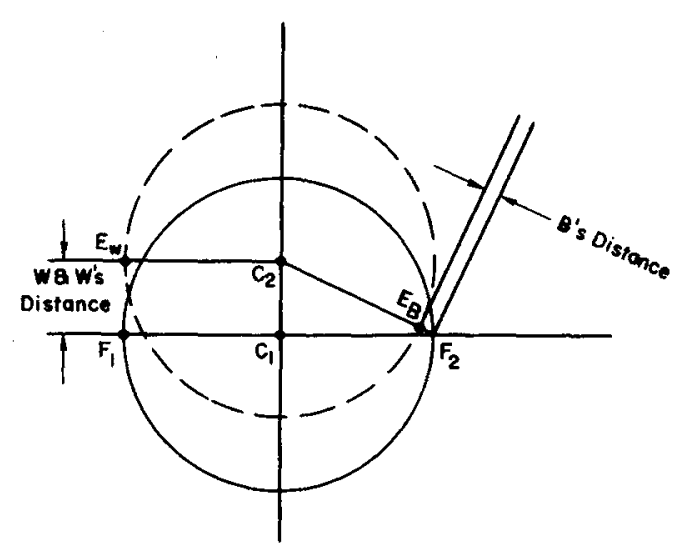

Figure 1. Calculation of target displacements and contour angles. $F_{1}$ : Fixation position of eye on target at start of trial. $F_{2}$ : Fixation position at end of trial. $C_{1}$ : Center of stimulus circle at start of trial. $C_{2}$ : Center at end of trial. $E_{w}$ : Final position for the edge of the target in the calculations reported in Whipple and Wallach's Table 1. $E_{7}$ : Edge of the target nearest $F_{2}$, used to recalculate displacement ratios (see text).

Figure 1). A similar analysis can be applied to Whipple and Wallach's other stimulus movement conditions.

The result of these calculations is that the part of the circle nearest the fovea is actually closer to the center of the fovea in the orthogonal condition $\left(.41^{\circ}\right)$ than it is in the congruent condition $\left(.56^{\circ}\right)$, despite the fact that the thresholds calculated by Whipple and Wallach showed the reverse relationship $(25 \%$ and $8 \%$, respectively). In this analysis, the large difference in thresholds between Whipple and Wallach's congruent conditions and their conditions with orthogonal components disappears and even reverses slightly. The smaller distance from the expected contour position to the actual position found in the orthogonal trials is always accompanied by a change in the orientation of this closest contour from its expected value, however. This change in orientation may serve to reduce the threshold values from those found in the congruent conditions to those found in the orthogonal ones: even though the displacement discrepancy is smaller, the angle discrepancy provides additional information to inform the subject of a noncongruent displacement. (This angle is not the same as the angle $\theta$ in Whipple and Wallach's analysis.)

The above analysis brings Whipple and Wallach's qualitative result into approximate agreement with the congruent vs. orthogonal data of Mack (1970) and of Stark et al. (1976), which show no difference in threshold under the two conditions. Remaining differences among these papers can be attributed to differences in stimuli and in response measure. The new analysis turns up a larger and more consistent quantitive discrepancy between the results of Whipple and Wallach and those of others, however, for 
Whipple and Wallach obtained recalculated displacement ratios of less than $10 \%$ under all conditions while all of the seven papers cited at the beginning of this note found threshold displacement ratios of more than twice this amount. The most consistent way of reconciling the Whipple and Wallach thresholds with the others in the literature is to assume that the technical factors listed above, and especially the long time constant of amplification used on some trials, lowered their threshold values below those commonly found.

We conclude that human subjects are relatively insensitive to target movements during saccades, for their displacement thresholds rise from a few minutes of arc during fixation to over one-fifth of saccade amplitude during saccades. Saccadic suppression of displacement remains an important supplement to the frame-of-reference computation mechanism.

The origin of this effect is still unknown, however. Recent experiments in our laboratory show that it is not a result of attentional or criterion shifts during saccades, for the threshold displacement ratio remains high when a criterion-free two-alternative forced choice method is used. Figure 2 shows a plot of these data using a display format similar to that used in Whipple and Wallach's Figure 1, Condition 5. In this experiment, an on-line digital computer monitored horizontal eye movements measured with a paired-photocell system. The subject began a trial by saccading horizontally from one side of a $20^{\circ}$ target to the other and back; the computer responded with an auditory signal and repeated the trial if either saccade was accompanied by a blink, was too small, or showed abnormal dynamics. It also displaced the target horizontally in a square-wave step during one of the two saccades, and the subject pressed a button corresponding to the saccade on which the displacement had occurred. Subjects were required to guess if they were unsure. Target displacement amplitude varied from $.5^{\circ}$ to $4^{\circ}$ in $.5^{\circ}$ steps, and was adjusted

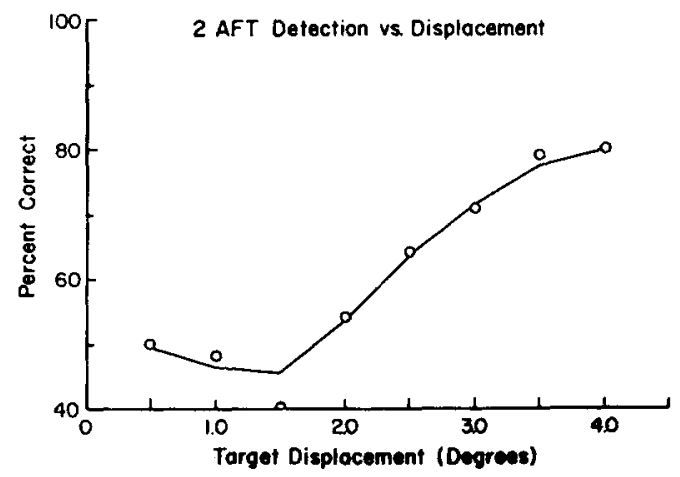

Figure 2. Percent correct detection of a target displaced $17 \mathrm{msec}$ after initiation of a saccade as a function of target displacement magnitude, for a $20^{\circ}$ eye movement. The line through the data points was smoothed by the function $B^{\prime}=(A+2 B+C) / 4$. The graph is based on pooled data from 300 trials in two subjects. on-line by a modified staircase procedure based on the subject's history of accuracy during the run. In this experiment, the $80 \%$ threshold was about $4.0^{\circ}$, corresponding to a $20 \%$ displacement ratio using the definitions of Whipple and Wallach, and confirming the higher displacement ratios in the literature. Other experiments in our laboratory (Bridgeman \& Lewis, 1976; Bridgeman, Lewis, Heit, \& Nagle, in press) have shown that the increase in threshold for displacements during saccades does not extend to a pointing measure of visual direction, limiting the generality of the effect to symbolic (nonisomorphic) measures of target position.

\section{REFERENCE NOTES}

1. Whipple, W. R. Personal communication, December 1, 1978.

\section{REFERENCES}

Bahill, A. T., Ciuffreda, K. H., Kenyon, R. V., \& Stark, L. Dynamic and static violations of Hering's law of equal innvervation. American Journal of Optometry and Physiological Optics, 1976, 53, 786-796.

BaHILL, A. T., \& STARK, L. Neurological control of horizontal and vertical components of oblique saccadic eye movements. Mathematical Biosciences, 1975, 27, 287-298.

Bahill, A. T., \& StaRK, L. Oblique saccadic eye movements: Independence of horizontal and vertical channels. Archives of Ophthalmology, 1977, 95, 1258-1261.

BEELER, G. Visual threshold changes resulting from spontaneous saccadic eye movements. Vision Research, 1967, 7, 769-775.

Bridgeman, B., Hendry, D., \& Stark, L. Failure to detect displacement of the visual world during saccadic eye movements. Vision Research, 1975, 15, 719.722.

BRIDGEMAN, B., \& Lewis, S. How the eye knows where the world is. Neurosciences Abstracts, 1976, 2, 276.

Bridgeman, B., Lewis, S., Heit, G., \& Nagle, M. The relationship between cognitive and motor-oriented systems of visual position perception. Journal of Experimental Psychology: Human Perception and Performance, in press.

BRUNe, F., \& Lückıng, C. Oculomotorik, Bewegungswahrnehmung und Raumkonstanz der Sehdinge. Der Nervenarzt, $1969,40,413-421$.

HERING, E. The theory of binocular vision (B. Bridgeman, trans., and B. Bridgeman \& L. Stark, Eds.). New York: Plenum, 1977. (Originally published, 1868.)

Leibowitz, H., Johnson, C., \& Isabelle, E. Peripheral motion detection and refraction error. Science, 1972, 77, 1207-1208.

MACK, A. An investigation of the relationship between eye and retinal image movement in the perception of movement. Perception \& Psychophysics, 1970, 8, 291-298.

Mack, A.. Fendrich, R., \& Pleune, J. Adaptation to an altered relation between retinal image displacements and saccadic eye movements. Vision Research, 1978, 18, 1321-1328.

STARK, L., KONG, R., SChWartz, S., HENDRY, D., \& Bridgeman, B. Saccadic suppression of image displacement. Vision Research, 1976, 16, 1185-1187.

WALlACH, H., \& LEWIS, C. The effect of abnormal displacement of the retinal image during eye movements. Perception \& Psychophysics, 1966, 1, 25.29.

WhIPPLE. W. R., \& Wallach, H. Direction-specific motion thresholds for abnormal image shifts during saccadic eye movement. Perception \& Psychophysics, 1978, 24, 349-355.

(Received for publication January 9, 1979; accepted January 10, 1979.) 